\title{
Empowerment of women in Science: Myth or Reality
}

Hortensia M. Rodríguez Cabrera

Recent history shows that nothing has been given to women. Every step taken in the way of the tireless task of achieving than what is fair has been synonymous of strikes, mobilization and daring, in addition to countless repressive demonstrations against the pioneers in the development of labor and social policies for the workers. Latest studies show that while political equality could be achieved in just over a century, projections for women economic empowerment, from salaries to financial control over assets, are two centuries. ${ }^{1}$ However, what about women in STEM (Science, Technology, Engineering, and Mathematics)? Taking into account that it is precisely in this area where future jobs and sustainable growth appear to be found, the search for solutions to the gender gap in STEM is more relevant. Although in recent years the participation of women in the scientific-technological professional environment has increased, there is still an under-representation. We have been the great ones forgotten or omitted, but women have also had, we have, and we will have much to say in terms of science.

The Institute for Statistics of UNESCO ${ }^{2}$ provided data on the positions that women occupy in science, through research carried out worldwide. The statistics concluded that, at the global level, only $28 \%$ of scientific research positions are occupied by women. Although the number of women who enroll in the university to study careers such as Natural Sciences, Engineering, Technology, Social Sciences, Humanities, and Agricultural Sciences is increasing, it is still pending in the world of science -as well as in the field of corporations- that women constitute the highest positions from researcher to project leaders. ${ }^{3}$ Despite the advances in certain senses, at a global level, there are still enough inequalities. An example of this is that, although $20 \%$ of the engineering graduates are women, they only represent $11 \%$ of the active engineers in the job market. Of more significant impact, ten years after graduating, only 3 out of 100 continue to work in fields related to STEM. ${ }^{4}$

A report prepared by the UNESCO (Education sector) in 2017, for girls and women around the world, advocates for their rights to quality education, full life, and a better future, but also delves into the factors that affect this gender gap. In particular, the factors include issues such as stereotypes, family beliefs, or the sociocultural context. Likewise, and contrary to what is defended, the document discards the influence of biological factors. ${ }^{5}$

Moreover, numerous studies report that women in the STEM fields publish less, are paid less for their research and do not progress to the level of men in their careers. However, there are still very few data at the international level that show the real scope of these differences. Faced with this reality, it is clear that it is necessary to generate and promote measures that contribute to minimize/eliminate the gender gap in STEM.

The fact that one of the Sustainable Development Goals is "Gender Equality" shows us the importance of addressing gender gaps in any field and, especially, in younger generations, and above all, with girls. In this sense, I believe that positive discrimination will not be the path that takes us to occupy the place that belongs to us by right in science, and not to which we have been relegated/made invisible in so many years of patriarchal society. Any initiative or measures to reduce gender inequality will inevitably go through education with a gender perspective to the whole society, but especially to our children.

Educational systems and schools play a central role in determining girls' interest in STEM subjects, and in providing equal opportunities to access and benefit from quality STEM education. Besides, projects such as TeachHer, ${ }^{6}$ which places the focus on the development of a STEM faculty capable of working and promoting these disciplines from a gender perspective, allowed to provided teachers with STEM training that allows the development of skills characteristic of these disciplines, working them in an equal, attractive and empowering way. Another positive example is Women In Science (WiSci), ${ }^{7}$ a STEM summer camp for high school girls from around the world. The initiative focuses on empowering adolescents to pursue careers in the STEM field, fostering intercultural learning and the development of ideas that promote global social well-being.

Despite the remarkable advances made in recent decades, education is not universally available, and gender inequalities are widespread, often to the detriment of girls. Complex and interrelated cultural and socioeconomic factors affect not only the opportunities for girls to attend school, but also the quality of education they will receive, the studies they will be able to follow and, ultimately, their careers and life trajectories. One of the biggest concerns worldwide is the low participation and performance of girls in science, technology, engineering, and mathematics (STEM) disciplines. Women must grow up, especially in the scientific world, assuming without fear of failure those roles until now mostly reserved for men. We must become principal investigators, heads of research groups, directors and why not, ministers of science and education or presidents. I advocate making more aggressive policies than those that have been done so far so that all these women who are formed in STEM occupy the highest positions and receive enough

\section{El empoderamiento de las mujeres en la ciencia: mito o realidad}

La historia reciente muestra que nada se le ha regalado a las mujeres. Cada paso dado en la incansable tarea de lograr lo que es justo ha sido sinónimo de huelga, movilización y audacia, además de innumerables manifestaciones represivas contra los pioneros en el desarrollo de políticas laborales y sociales para los trabajadores. Los estudios más recientes revelan que si bien la igualdad política se puede lograr en poco más de un siglo, las proyecciones para el empoderamiento económico de las mujeres, desde los salarios hasta el control financiero sobre los activos, serán dos siglos. ${ }^{1}$ Sin embargo, ¿Qué pasa con las mujeres en STEM (Ciencia, Tecnología, Ingeniería y Matemáticas por sus siglas en inglés)? Teniendo en cuenta que es precisamente en esta área donde se encuentran los empleos futuros y el crecimiento sostenible, la búsqueda de soluciones a la brecha de género en STEM cobra relevancia. Aunque en los últimos años ha aumentado la participación de 
las mujeres en el entorno profesional científico-tecnológico, todavía están subrepresentadas. Hemos sido las grandes olvidadas u omitidas, pero las mujeres también tuvimos, tenemos, y tendremos mucho que decir en términos de ciencia.

El Instituto de Estadísticas de la UNESCO ${ }^{2}$ proporcionó datos sobre las posiciones que ocupan las mujeres en la ciencia, a través de investigaciones realizadas en todo el mundo. Las estadísticas concluyeron que, a nivel mundial, solo el $28 \%$ de los puestos de investigación científica están ocupados por mujeres. Aunque el número de féminas que se matriculan en la universidad para estudiar carreras como Ciencias Naturales, Ingeniería, Tecnología, Ciencias Sociales, Humanidades y Ciencias Agrícolas está aumentando, todavía está pendiente en el mundo de la ciencia, así como en el campo de las corporaciones, que las mujeres asciendan a las posiciones más altas desde investigadoras a líderes de proyectos. ${ }^{3} \mathrm{~A}$ pesar de los avances, a nivel global todavía hay desigualdades. Un ejemplo de esto es que, aunque el $20 \%$ de los graduados en ingeniería son mujeres, solo representan el $11 \%$ de los ingenieros activos en el mercado laboral. De mayor impacto resulta que diez años después de graduarse, solo 3 de cada 100 continúan trabajando en campos relacionados con STEM. ${ }^{4}$

Un informe preparado por la UNESCO (sector de la educación) en 2017, para niñas y mujeres de todo el mundo, aboga por sus derechos a una educación de calidad, a una vida plena y a un futuro mejor, pero también profundiza en los factores que afectan esta brecha de género. En particular, los factores incluyen temas como los estereotipos, las creencias familiares o el contexto sociocultural. Asimismo, y en contra de lo que se defiende, el documento descarta la influencia de factores biológicos. ${ }^{5}$

Además, numerosos estudios informan que las mujeres en los campos de STEM publican menos, les pagan menos por sus investigaciones y no progresan al nivel de hombres en sus carreras. No obstante, todavía hay muy pocos datos a nivel internacional que muestran el alcance real de estas diferencias. Frente a esta realidad, es clara la necesidad de generar y promover medidas que contribuyan a minimizar / eliminar la brecha de género en STEM.

El hecho de que uno de los Objetivos de Desarrollo Sostenible sea "Igualdad de Género" reafirma la importancia de abordar las brechas de género en cualquier campo, especialmente en las generaciones más jóvenes y, sobre todo, con las niñas. En este sentido, creo que la discriminación positiva no será el camino que nos lleve a ocupar el lugar que nos pertenece por derecho en la ciencia, y no al que hemos sido relegadas / invisibilizadas en tantos años de sociedad patriarcal. Cualquier iniciativa o medida para reducir la desigualdad de género pasará inevitablemente por la educación con una perspectiva de género de toda la sociedad, pero muy especialmente a nuestros niños.

En este sentido, los sistemas educativos y las escuelas desempeñan un papel central en la determinación del interés de las niñas en las materias STEM y en brindar igualdad de oportunidades para acceder y beneficiarse de una educación STEM de calidad. Además, proyectos como TeachHer, ${ }^{6}$ que se centran en el desarrollo de profesionales STEM capaces de trabajar y promover estas disciplinas desde una perspectiva de género, permitieron a los docentes capacitarse en STEM y permitirles el desarrollo de habilidades propias en estas disciplinas. Inculcándoles el trabajo de forma igualitaria, atractiva y empoderadora. Otro ejemplo positivo es Women in Science (WiSci), ${ }^{7}$ un campamento de verano STEM para niñas de secundaria de todo el mundo. La iniciativa se centra en capacitar a los adolescentes para que sigan carreras en este campo, fo- mentando el aprendizaje intercultural y el desarrollo de ideas que promuevan el bienestar social global.

A pesar de los notables avances logrados en las últimas décadas, la educación no está disponible de manera universal, y las desigualdades de género están generalizadas, a menudo en detrimento de las niñas. Los factores culturales y socioeconómicos complejos e interrelacionados afectan no solo las oportunidades para que las niñas asistan a la escuela, sino también a la calidad de la educación que recibirán, los estudios que podrán seguir y, en última instancia, sus carreras y trayectorias de vida. Una de las mayores preocupaciones a nivel mundial es la baja participación y el rendimiento de las niñas en las disciplinas de ciencia, tecnología, ingeniería y matemáticas (STEM). Las mujeres deben crecer, especialmente en el mundo científico, asumiendo sin temor a fallar esos roles hasta ahora mayormente reservados para los hombres. Debemos convertirnos en investigadoras principales, jefas de grupos de investigación, directoras y, por qué no, en ministras de ciencia y educación o presidentes. Abogo por hacer políticas más agresivas que las que se han hecho hasta ahora para que todas estas mujeres que se forman en STEM ocupen los puestos más altos y reciban suficiente dinero para investigar.

money to investigate.

\section{References}

1. https://www.bbc.com/mundo/noticias-46638119?ocid=socialflow_twitter

2. (http://uis.unesco.org/)

3. http://uis.unesco.org/sites/default/files/documents/fs51-women-in-science-2018-en.pdf

4. https://observatorio.profuturo.education/brecha-de-genero-enstem/

5. Cracking the code: girls' and women's education in science, technology, engineering and mathematics (STEM) https://unesdoc. unesco.org/ark:/48223/pf0000253479

6. https://observatorio. profuturo.education/blog/2018/05/22/promoviendo-politicas-de-igualdad-de-genero-desde-el-fomento-de-las-vocaciones-steam-teachher/

7. https://observatorio. profuturo.education/blog/2018/05/04/ el-imprescindible papel-de-la-mujer-en-la-ciencia-wisci-girlssteam-camp/ 